\title{
A Simple Proof Of The Non Existence Of The Centre Of A Parabola
}

\section{Soham Dasgupta}

(Ex. PGT (Math) at Kendriya Vidyalaya No.2 Saltlake, Kolkata, W.B, INDIA)

\begin{abstract}
The study about the centre of a conic section is always a major part in basic coordinate geometry. This study consists of the existence and finding of the centre of a given conic. In many books of coordinate geometry we usually notice a statement that the centre of a parabola is at infinity. But this statement is actually wrong. The correct statement should be there exist no centre of a parabola. Because 'at infinity' means there exist but we can't find it. Here we had proved this simple thing using very simple algebraic calculations. We had also enclosed the long calculative classical method of it as defined in some well known coordinate geometry books.
\end{abstract}

Keywords: Centre,Chord,Conic,Parabola, central and non-central conics.

Chord: If $\zeta$ be a planer curve and A \& B are two distinct points over it then the line segment joining A\&B, i.e $\mathrm{AB}$ is called a chord.

Centre: The centre of a conic section is a point over the plane such that all chords of the conic which passes through it are bisected at that point.

Now for any central curve (i.e curves having centre) we will consider the centre is at origin $(0,0)$. That is using rigid motion we will move any central curves centre at $(0,0)$.

\section{Theorem: Parabola is a non central conic.}

Proof: As the general equation of a parabola is $(y-k)^{2}=4 a(x-h)$, let it have a centre and its at $(0,0)$. Now if $\left(\mathrm{x}_{1}, \mathrm{y}_{1}\right) \&\left(\mathrm{x}_{2}, \mathrm{y}_{2}\right)$ are the ends of a chord of the parabola passing through the origin then obviously those points are linearly dependent. Again by the definition of centre we get that $\left(\mathrm{x}_{2}, \mathrm{y} 2\right)=(-\mathrm{x} 1,-\mathrm{y} 1)$.

Thus satisfying the equation of parabola using the points we get,

$(\mathrm{y} 1-\mathrm{k})^{2}=4 \mathrm{a}(\mathrm{x} 1-\mathrm{h})$

-(i) and $\left(-y_{1}-k\right)^{2}=4 a\left(-x_{1}-h\right)$, i.e $\left(y_{1}+k\right)^{2}=-4 a\left(x_{1}+h\right)$

Now subtracting eq.(ii) from (i) we get, $4 \mathrm{y}_{1} \mathrm{k}=-8 \mathrm{ax} 1$

Now let $\mathrm{y}=\mathrm{mx}$ be the line on which the $\left(\mathrm{x}_{1}, \mathrm{y}_{1}\right)$ and $\left(\mathrm{x}_{2}, \mathrm{y}_{2}\right)$ are situated. Thus $\mathrm{y}_{1}=\mathrm{mx}_{1}$ and putting it in eq. (iii) we get

$$
\begin{gathered}
4 \mathrm{mx} 1=-8 \mathrm{ax} 1 \\
\text { Or, } 4 \mathrm{x}_{1}(2 \mathrm{a}+\mathrm{mk})=0
\end{gathered}
$$

So, either $\mathrm{x}_{1}=0$ or $(2 \mathrm{a}+\mathrm{mk})=0$

But if $\mathrm{x}_{1}=0$ then $\left(\mathrm{x}_{1}, \mathrm{y}_{1}\right)=\left(\mathrm{x}_{2}, \mathrm{y}_{2}\right)=(0,0)$, which is impossible.

Now for the case of $2 a+m k=0$, notice that here $m$ is arbitrary but a and $k$ are constant. That is if we take another line say $\mathrm{y}_{\mathrm{a}}=\mathrm{m}_{0} \mathrm{x}$, where $\mathrm{m}_{0} \neq \mathrm{m}$, and which intersects the parabola at $\left(\mathrm{x}_{3}, \mathrm{y}_{3}\right) \&\left(\mathrm{x}_{4}, \mathrm{y}_{4}\right)$, then also we will have the similar eq. $2 \mathrm{a}+\mathrm{m}_{0} \mathrm{k}=0$.

So this case is also impossible, therefore we arrived at the conclusion that Parabola has no centre. 
To find the coordinates of the centre of a conic section.

Let

$$
a x^{2}+2 h x y+b y^{2}+2 g x+2 f y+c=0
$$

be the equation of the conic and let $\left(x^{\prime}, y^{\prime}\right)$ be its centre.

Transferring the origin to the point $\left(x^{\prime}, y^{\prime}\right)$ by taking the axes. Through the point $\left(x^{\prime}, y^{\prime}\right)$ parallel to the original axes and substituting $\mathrm{X}+\mathrm{x}^{\prime}$ for $\mathrm{x}$ and $\mathrm{Y}+\mathrm{y}^{\prime}$ for $\mathrm{y}$, equation (1) will become

$$
a\left(X+x^{\prime}\right)^{2}+2 h\left(X+x^{\prime}\right)\left(Y+y^{\prime}\right)+b\left(Y+y^{\prime}\right)^{2}+2 g\left(X+x^{\prime}\right)+2 f\left(Y+y^{\prime}\right)+c=0
$$

In this equation the coefficients of $\mathrm{X}$ and $\mathrm{Y}$ will both be zero, if $\mathrm{x}$ and $\mathrm{y}$ be so chosen that

$$
\begin{aligned}
& a x^{\prime}+h y^{\prime}+g=0 \\
& \text { and } \quad h x^{\prime}+b y^{\prime}+f=0 .
\end{aligned}
$$

Thus equation (2) becomes

$$
\mathrm{aX}^{2}+2 \mathrm{hXY}+\mathrm{bY}^{2}+\mathrm{c}^{\prime}=0
$$

where

$$
c^{\prime}=a x^{\prime 2}+2 h x^{\prime} y^{\prime}+b y^{\prime 2}+2 g x^{\prime}+2 f y^{\prime}+c
$$

By solving equations (3) and (4) simultaneously, the centre of the conic can be obtained as

$$
\text { (fh-bg / ab-h², gh-af / ab-h }{ }^{2} \text { ) }
$$

Now we know that in case of parabola $a b-h^{2}=0$, thus such point can't exist for parabola.

Conclusion: Thus the correct statement should be there exist no centre of a parabola. Because 'at infinity' means there exist a centre but it's impossible to find that.

\section{References:}

1. P. Jain, K. Ahmad - A Textbook Of Analytical Geometry Of Two Dimensions-New Age (1996)

2. (Dover Books on Mathematics) Luther Pfahler Eisenhart - Coordinate Geometry-Dover Publications (2005) 